\begin{tabular}{|c|c|}
\hline Title & A symmetric Total Synthesis of Brasilicardins \\
\hline Author(s) & Yoshimura, Fumihiko; Itoh, Ryusei; Torizuka, Makoto; Mori, Genki; Tanino, Keiji \\
\hline Citation & $\begin{array}{l}\text { A ngewandte Chemie International Edition, 57(52), 17161-17167 } \\
\text { https://doi.org/10.1002/anie.201811403 }\end{array}$ \\
\hline Issue Date & $2018-12-21$ \\
\hline Doc URL & http://hdl.handle.net/2115/76353 \\
\hline Rights & $\begin{array}{l}\text { This is the peer reviewed version of the following article:A ngewandte Chemie (International ed.) 57(52) December } 21 \\
\text { 2018, pp.17161-17167 which has been published in final form at https://doi.org/10.1002/anie.201811403. This article } \\
\text { may be used for non-commercial purposes in accordance with Wiley Terms and Conditions for U se of Self-A rchived } \\
\text { V ersions. }\end{array}$ \\
\hline Tyре & article (author version) \\
\hline File Information & A ngew. Chem. Int. Ed.57-52_17161-17167.pdf \\
\hline
\end{tabular}

Instructions for use 


\title{
Asymmetric Total Synthesis of Brasilicardins
}

\author{
Fumihiko Yoshimura, ${ }^{*[a, c]}$ Ryusei Itoh, ${ }^{[b]}$ Makoto Torizuka, ${ }^{[b]}$ Genki Mori, ${ }^{[a]}$ and Keiji Tanino* ${ }^{[a]}$
}

\begin{abstract}
Brasilicardins are bacterial diterpenoid natural products that display highly potent immunosuppressive activity; therefore, they are considered to be promising immunosuppressant drug candidates. Structurally, they can be described as hybrids of terpenoids, amino acids, and saccharides, and share a characteristic highly strained anti-syn-anti-fused perhydrophenanthrene terpenoid scaffold (ABC-ring system) with two quaternary asymmetric carbon atoms. Herein, we describe our design of the unified and stereoselective total synthesis of all four brasilicardins based on the strategic use of an intramolecular conjugate addition. The ABC-ring system was initially constructed with high stereocontrol by novel intramolecular conjugate additions of Weinreb amides and in situgenerated (Z)-vinyl copper species. The late-stage common intermediate was subjected to stereoselective installation of the amino acid component, followed by introduction of the saccharide unit via glycosylation to accomplish the total synthesis of brasilicardins A-D. Our synthesis offers opportunities to synthesize various brasilicardin analogues for biological and pharmacological investigations.
\end{abstract}

Brasilicardins A-D (1-4) are bacterial diterpenoid natural products isolated from the cultured broth of the actinomycete Nocardia brasiliensis IFM 0406, which exhibit diverse biological activities (Figure 1). ${ }^{[1-3]}$ Among these congeners, brasilicardin A (1) displays potent immunosuppressive activity $\left(\mathrm{IC}_{50}, 0.05 \mathrm{nM}\right)$. Although the mechanism of the immunosuppressive action of 1 has not been clarified in detail, it has been suggested that it is induced by amino acid deprivation via the inhibition of the amino acid transporter system L. ${ }^{[4]}$ Currently used immunosuppressive clinical agents such as tacrolimus and cyclosporin A often cause side effects such as nephrotoxicity and arterial hypertension; therefore, an alternative to them is desired. In this context, 1 is thus considered to be a promising drug candidate. Thus, 1 has been studied with keen interest, particularly in to the context of the development of new immunosuppressive drugs. ${ }^{[5,6]}$ However, further biological and pharmacological studies of 1 have not been explored because of the limited availability of 1 from natural

[a] Prof. Dr. F. Yoshimura, G. Mori, Prof. Dr. K. Tanino Department of Chemistry, Faculty of Science Hokkaido University

Sapporo 060-0810, Japan

E-mail: ktanino@sci.hokudai.ac.jp (K.T.)

[b] R. Itoh, M. Torizuka

Graduate School of Chemical Sciences and Engineering

Hokkaido University

Sapporo 060-0810, Japan

[c] Present Address: Prof. Dr. F. Yoshimura

School of Pharmaceutical Sciences

University of Shizuoka

52-1 Yada, Suruga-ku, Shizuoka 422-8526, Japan

E-mail: fumi@u-shizuoka-ken.ac.jp (F.Y.)

Supporting information for this article is given via a link at the end of the document. sources; therefore, efficient chemical syntheses of 1 and its derivatives and simplified analogues are required to aid further studies.
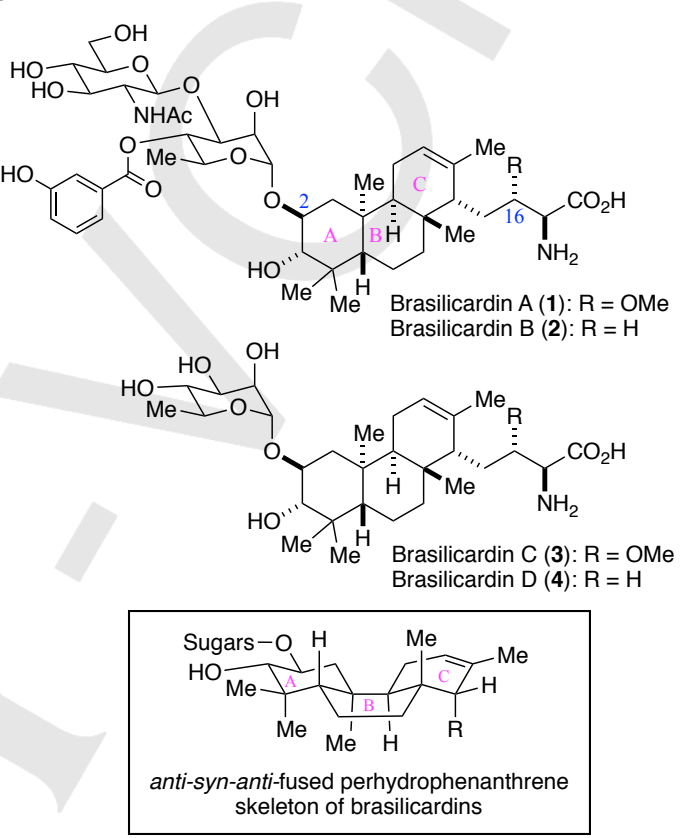

Figure 1. Structures of brasilicardins.

Structurally, brasilicardins A-D (1-4) share a unique and highly strained anti-syn-anti-fused perhydrophenanthrene diterpenoid carbon scaffold (i.e., the ABC-ring) containing two angular methyl groups with the B-ring boat conformer (Figure 1). To this scaffold, different amino acid and saccharide units are attached.

The combination of their distinctive biological and pharmacological properties and the novel chemical structures has made brasilicardins extremely attractive targets for synthetic organic chemists. ${ }^{[6-9]}$ However, their complex and unique threedimensional structure has hampered their chemical synthesis. Despite considerable synthetic efforts having been undertaken for over twenty years, the sole successful total synthesis was the de novo construction of 1 and $\mathbf{3}$ by Anada, Hashimoto, and coworkers in $2017^{[10]}$ which involved sequential functionalization of the Wieland-Miescher ketone derivative and a Diels-Alder reaction/reductive angular methylation sequence ${ }^{[7]}$ (Scheme 1a). Herein, we report a novel and unified approach that enables the total synthesis of all brasilicardins (1-4), including the first total synthesis of $\mathbf{2}$ and $\mathbf{4}$.

From a synthetic perspective, the challenges posed by brasilicardins (1-4) are as follows: (1) development of a stereoselective synthetic methodology for the unique carbocyclic framework (the $A B C$-ring), which represents the most important issue in the synthesis, (2) construction of the amino acid 
a Anada-Hashimoto approach

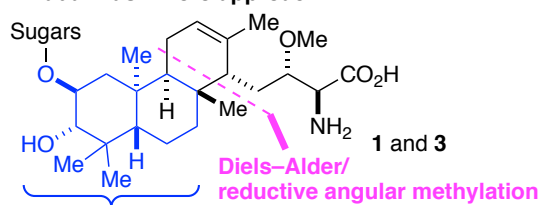

From Wieland-Miescher ketone derivative

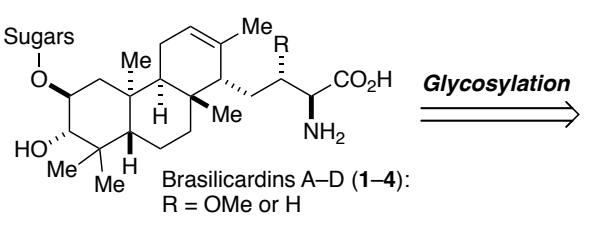

b Our key technology

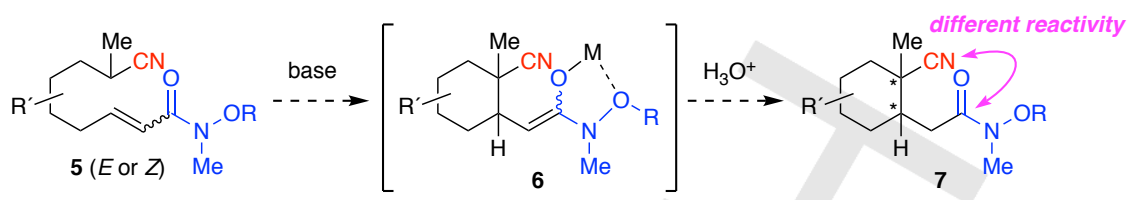

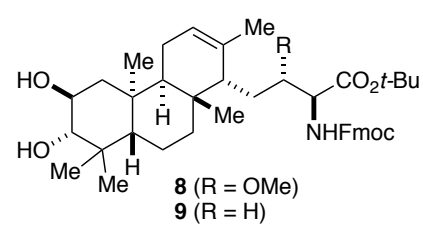
$9(\mathrm{R}=\mathrm{H})$

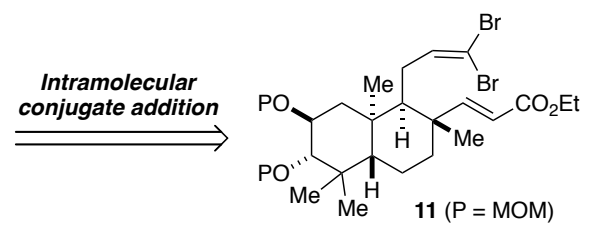

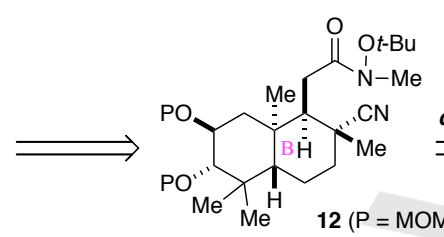

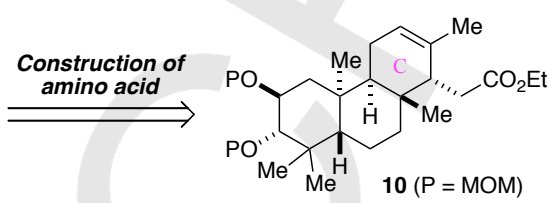

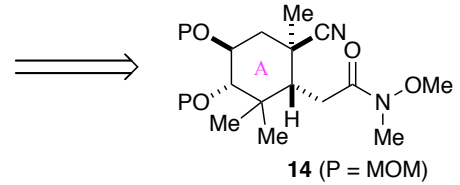

Intramolecular conjugate addition<smiles>CON(OC)C(=O)/C=C/C(C)(C)C([Pb])C(CC(C)(C)C)C(=O)P</smiles>

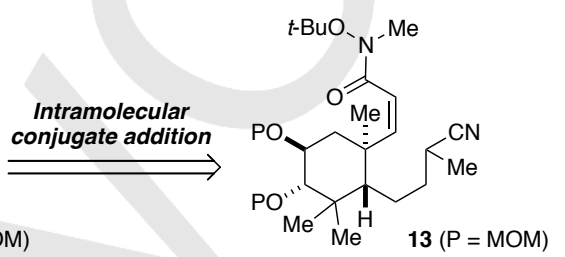

Scheme 1. Background and synthetic plan.

component, and (3) stereoselective installation of the sugar moiety.

Aiming to obtain in-depth structure-activity relationships (SAR) in future for understanding the mode of action of 1 at the molecular level, we employed a tactic wherein each ring of the carbocyclic framework as well as the amino acid and saccharide components would be constructed in a step-by-step manner. While a biomimetic polyene cyclization affording the above framework could have been a potential strategy, a previous study revealed that such an approach was extremely difficult. ${ }^{[11]}$ As one of the key steps, we designed an intramolecular conjugate addition (Michael addition) of $\alpha, \beta$-unsaturated $N$-methoxy- $N$ methylamides (commonly named Weinreb amides ${ }^{[12]}$ ) bearing an alkanenitrile side chain (Scheme $1 b ; 5 \rightarrow 7$ ). Despite the poor reactivities of both $\alpha, \beta$-unsaturated amides and $\alpha$-cyano carbanions generated from simple non-activated alkanenitriles as Michael acceptors and Michael donors, respectively, ${ }^{[13,14]}$ we expected that the Michael addition would proceed smoothly because the putative and stable chelated intermediate 6 formed upon the addition would prevent unfavored reversible retroadditions. In this connection, we previously reported another approach for preventing similar retro-additions by trapping the intermediate as the corresponding ketene silyl acetal in an intramolecular Michael addition of $\alpha, \beta$-unsaturated lactones. ${ }^{[15]}$ In addition, if such additions were to occur with facial discrimination, the sterically much less demanding cyano group would induce stereoselective cyclization, resulting in simultaneous construction of a contiguous quaternary-tertiary stereocenter. Moreover, the potential different reactivities between the sterically congested cyano group and Weinreb amide groups in the product 7 would facilitate the further transformations.

With the above considerations in mind, our retrosynthetic analysis of brasilicardins (1-4) is shown in Scheme 1c. The saccharide moiety was to be installed through regioselective glycosylation to the protected aglycons $\mathbf{8}$ or $\mathbf{9}$ at the final stage of the synthesis. Aglycons $\mathbf{8}$ or $\mathbf{9}$ were to be derived from ester 10 via construction of the amino acid component. Thus, we envisioned that the tricyclic core 10 could serve as an advanced intermediate for our unified synthesis. The requisite core 10 could be synthesized by a Michael addition-based strategy. To be more specific, 10 could be obtained from the $\alpha, \beta$-unsaturated ester 11 through our novel intramolecular Michael addition promoted by $\mathrm{Me}_{2} \mathrm{CuLi}^{[16]}$ The dibromide 11 could be synthesized from bicyclic compound 12 via homologation of the side chains. The B-ring could be constructed through an intramolecular nitrile Michael addition of $(Z)-\alpha, \beta$-unsaturated Weinreb amide 13 as mentioned above (see, Scheme $1 \mathrm{~b}$ ). Amide 13 would be derived from nitrile 14. The A-ring could be constructed by a similar Michael addition of $(E)-\alpha, \beta$-unsaturated Weinreb amide 15. This compound, in turn, could be accessed from commercially available 2,2-dimethylpropane-1,3-diol (16).

Our first objective focused on the stereoselective synthesis of the crucial precursor 15 for the first intramolecular nitrile Michael addition (Scheme 2). Monosilylation of 2,2dimethylpropane-1,3-diol (16) followed by Swern oxidation of the remaining alcohol afforded the corresponding aldehyde, which underwent one-pot Horner-Wadsworth-Emmons (HWE) olefination $^{[17]}$ to furnish $(E)-\alpha, \beta$-unsaturated ester 17 as the sole product. Asymmetric dihydroxylation of 17 by using the 


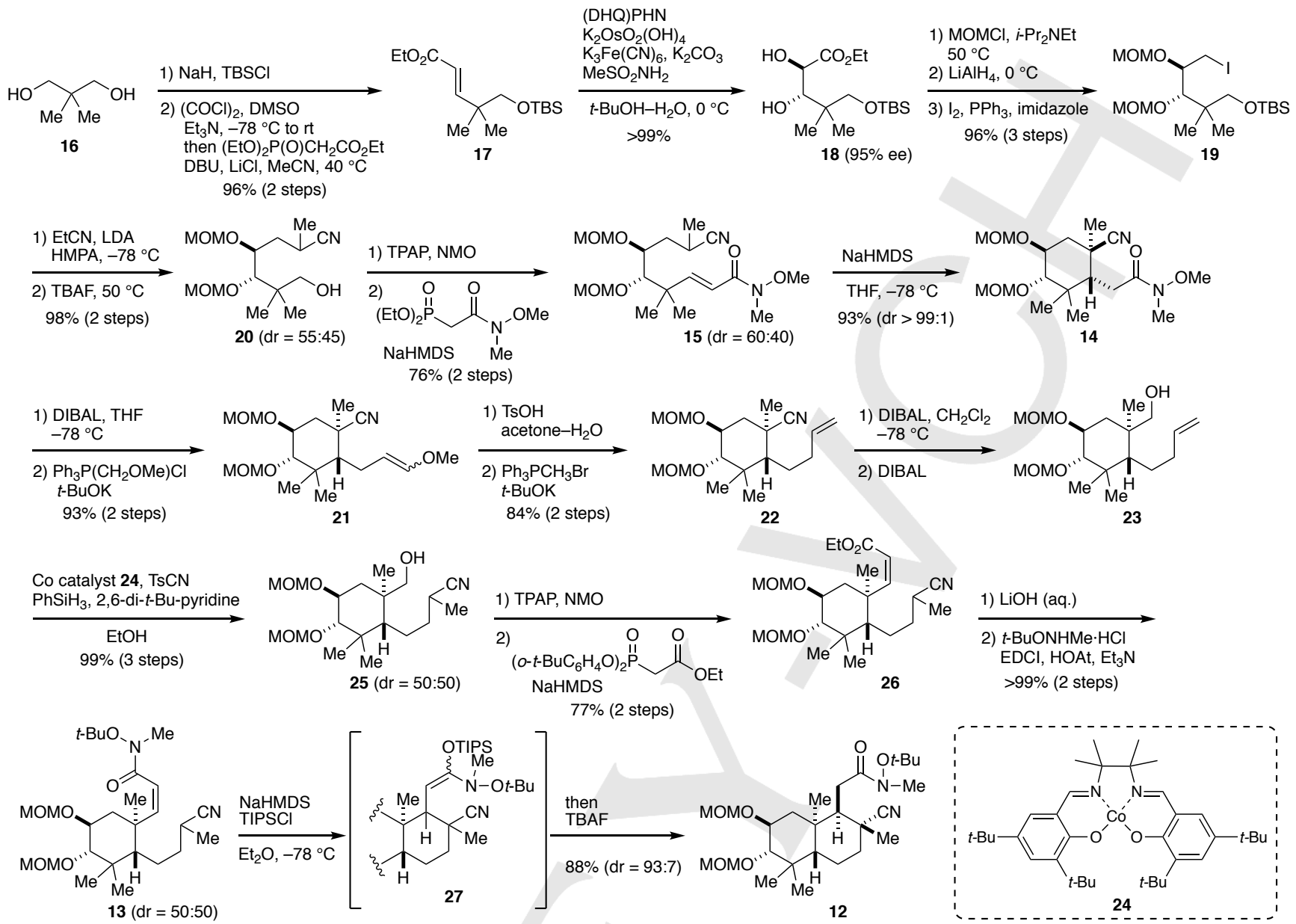

Scheme 2. Synthesis of the bicyclic intermediate 12. TBS = tert-butyldimethylsilyl, DBU = 1,8-diazabicyclo[5.4.0]undec-7-ene, MOM = methoxymethyl, LDA = lithium diisopropylamide, HMPA $=$ hexamethylphosphoramide, TPAP = tetrapropylammonium perruthenate, NMO = N-methylmorpholine oxide, NaHMDS = sodium bis(trimethylsilyl)amide, DIBAL = diisobutylaluminum hydride, $\mathrm{EDCl}=$ 1-ethyl-3-(3-dimethylaminopropyl)carbodiimide hydrochloride, $\mathrm{HOAt}=1$-hydroxy-7azabenzotriazole, TIPS = triisopropylsilyl, TBAF = tetrabutylammonium fluoride.

dihydroquinine-based 9'-phenanthryl ether ligand (DHQ)PHN ${ }^{[18,19]}$ quantitatively gave the optically active diol 18 with $95 \%$ ee. Protection of the diol in 18 with MOM groups, followed by reduction of the ethyl ester and iodination of the resulting alcohol, yielded alkyl iodide 19. Alkylation of 19 with deprotonated propionitrile and the subsequent desilylation afforded alcohol 20. This compound underwent oxidation with TPAP and HWE olefination to give $(E)-\alpha, \beta$-unsaturated Weinreb amide 15 as the substrate for an intramolecular Michael addition.

We then focused on the crucial intramolecular Michael addition of $\mathbf{1 5}$ for the formation of the A-ring (Scheme 2). In an extensive screening of reaction conditions, it was found that when a THF solution of 15 was treated with NaHMDS at $-78^{\circ} \mathrm{C}$, intramolecular Michael addition proceeded stereoselectively to furnish the desired product 14 in $93 \%$ yield as a single isomer. The stereochemistry of $\mathbf{1 4}$ was unambiguously determined by nuclear Overhauser effect (NOE) experiments (Supporting Information, Figure S1). The observed complete stereocontrol for 14 was suggested to arise as a consequence of chelation control, wherein the keteniminate and $\alpha, \beta$-unsaturated amide were both oriented equatorially in an antiparallel dipolar arrangement in the transition state model (Supporting Information, Scheme S1).

The successful realization of an intramolecular nitrile Michael addition led us to the next phase of the synthesis: construction of the B-ring (Scheme 2). Thus, chemoselective reduction of the Weinreb amide over the cyano group in 14 with DIBAL followed by one-carbon homologation of the resulting aldehyde by a Wittig reagent produced enol methyl ether 21 . The product $\mathbf{2 1}$ was further converted to terminal alkene $\mathbf{2 3}$ in four steps. Introduction of the cyano group into $\mathbf{2 3}$ was achieved by cobalt-catalyzed hydrocyanation $^{[20]}$ to give secondary nitrile 25 regioselectively. Oxidation of alcohol 25 with TPAP afforded the corresponding aldehyde, which underwent $Z$-selective HWE reaction ${ }^{[21,22]}$ to afford (Z)- $\alpha, \beta$-unsaturated ester 26 (ZIE > 99:1). Saponification of 26 and the subsequent amidation produced the modified Weinreb amide ${ }^{[23]}$ namely, $N$-tert-butoxy- $N$-methylamide 13.

The second intramolecular nitrile Michael addition of 13 was the crucial factor in the present synthesis; therefore, we explored the optimal reaction conditions for this step, focusing on the solvent, additives, and reaction temperature. We found that this 


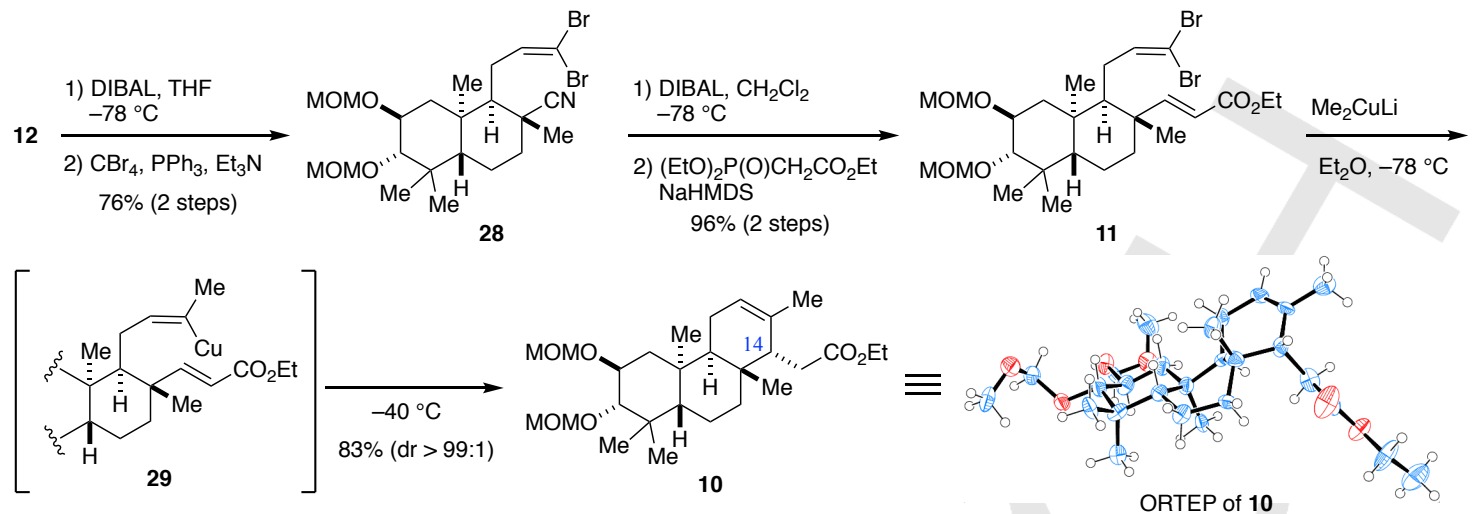

Scheme 3. Stereoselective synthesis of the tricyclic compound $\mathbf{1 0 .}$

addition occurred upon the treatment of a mixture of 13 and TIPSCl ${ }^{[15]}$ with NaHMDS in $\mathrm{Et}_{2} \mathrm{O}$ at $-78{ }^{\circ} \mathrm{C}$, which produced $\mathrm{O}$ silyl N,O-ketene acetal $\mathbf{2 7}$ in situ. Upon addition of TBAF to $\mathbf{2 7}$, the desired cyclization product 12 was obtained as a 93:7 inseparable mixture of diastereomeric products ( $88 \%$ yield). Importantly, in this cyclization, the use of the bulky O-tert-butyl Weinreb amide, (Z)-configuration of the olefin, and addition of TIPSCI were all indispensable to achieve a high level of stereocontrol at the newly formed stereocenters of the product (for comparison with the cyclization of other precursors, see Scheme $\mathrm{S} 2$ in the Supporting Information). The steric repulsions between the large substituents are likely to account for the stereochemical outcome (Supporting Information, Scheme S3). These key nitrile Michael additions (i.e., $15 \rightarrow \mathbf{1 4}$ and $\mathbf{1 3} \rightarrow \mathbf{1 2}$ ) were reliably performed on a gram scale, which demonstrated the significant synthetic utility of this methodology.

Having developed a method for the construction of the ABring including two quaternary asymmetric stereocenters based on the strategic nitrile Michael additions, we next focused on the third intramolecular Michael addition, which leads to the ABCring of brasilicardins (Scheme 3). To this end, Weinreb amide 12 was converted to 1,1-dibromoalkene 11, the requisite cyclization precursor, in a four-step sequence: (1) chemoselective DIBAL reduction of the Weinreb amide moiety in 12 to an aldehyde, (2) Corey-Fuchs olefination, wherein the minor diastereomer of 12 was separated, (3) reduction of the cyano group to an aldehyde, and (4) HWE olefination of the resulting aldehyde.

The third intramolecular Michael addition for constructing the C-ring proceeded smoothly under the reaction conditions used ${ }^{[16]}$ (Scheme 3): treating 11 with $\mathrm{Me}_{2} \mathrm{CuLi}$ (5 equiv) at $-78^{\circ} \mathrm{C}$ in $\mathrm{Et}_{2} \mathrm{O}$ for 30 min generated the (Z)-vinylcopper species 29 in situ. After the reaction mixture was warmed up to $-40{ }^{\circ} \mathrm{C}$ and then stirred at this temperature for $1 \mathrm{~h}$, the subsequent conjugate addition of 29 to the $\alpha, \beta$-unsaturated ester moiety proceeded smoothly in the one-pot system to furnish the tricyclic compound 10 with controlled stereochemistry at the $\mathrm{C} 14$ position ( $83 \%$ yield). The structure of $\mathbf{1 0}$ was verified by X-ray crystallographic analysis (Scheme 3). In this way, we established a novel method for the stereoselective construction of the anti-syn-anti-fused perhydrophenanthrene skeleton (the ABC-ring) by triple intramolecular Michael additions as the key steps.
On obtaining the tricyclic compound 10 , we directed our attention to installation of the appropriate amino acid portion to this skeleton (Scheme 4). Initially, we investigated installation of a $\beta$-methoxy amino acid for brasilicardins $A$ and $C$, which was succeeded by application of Rama Rao's procedure ${ }^{[24]}$ (Scheme 4a). Half-reduction of $\mathbf{1 0}$ followed by HWE reaction of the resulting aldehyde yielded $\alpha, \beta$-unsaturated tert-butyl ester 30 . Asymmetric dihydroxylation of $\mathbf{3 0}$ using (DHQ)PHN-ligand ${ }^{[18]}$ gave the desired diol $\mathbf{3 1}$ as a single diastereomer. Regioselective nosylation at the $\mathrm{C} 17$ alcohol of $\mathbf{3 1}$ produced mono-nosylate $\mathbf{3 2}$, and subsequent treatment of $\mathbf{3 2}$ with $\mathrm{NaN}_{3}$ afforded $\alpha$-azide 33 with complete inversion of the configuration. O-Methylation of 33 followed by reduction of the azide and protection of the resulting amine with a Fmoc group resulted in the formation of the protected amino acid of brasilicardins $\mathrm{A}$ and $\mathrm{C}$. Finally, removal of both MOM groups with $\mathrm{HCl}$ in methanol produced diol 8 . Stereochemistry of the amino acid component was verified by $\mathrm{X}$ ray crystallographic analysis after the conversion to the corresponding $p$-bromobenzamide derivative of 8 (Supporting Information, Figure S2).

On the other hand, installation of the amino acid component of brasilicardins B and D was performed by asymmetric alkylation of the chiral Schiff base ${ }^{[25]}$ as the key step (Scheme 4b). Thus, ethyl ester 10 was converted to iodide $\mathbf{3 4}$ through $\mathrm{LiAlH}_{4}$ reduction and iodination of the resulting alcohol. Alkylation of the readily available chiral Schiff base derived from glycine $\mathbf{3 5}$ with 34 proceeded smoothly when KHMDS was used as the base, providing the alkylation product $\mathbf{3 6}$ as the sole isomer. This compound was converted to diol $\mathbf{9}$ in three steps, i.e., removal of the chiral auxiliary group, Fmoc-protection of the resulting freeamine, and deprotection of the MOM groups. Stereochemistry of the amino acid portion was confirmed by the modified Mosher's method $^{[26]}$ (Supporting Information, Scheme S4).

The final task in the synthesis was the challenging glycosylation of aglycons 8 and 9 (Scheme 5). First, the glycosylation of 8 toward brasilicardin A (1) was explored (Scheme 5a). Although Anada and Hashimoto's group succeeded in glycosylation of the C3-protected aglycon of 1 with the trichloroacetimidate donor of the disaccharide unit, ${ }^{[8,10]}$ this procedure could not be applicable to diol 8 because the formation of complex mixtures including the inseparable C3 
a

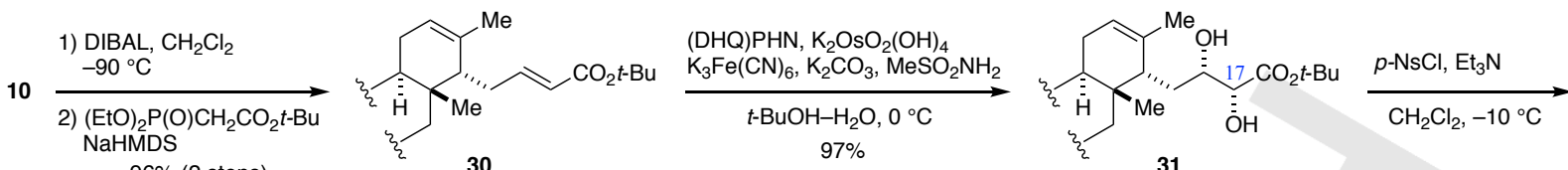
$96 \%$ (2 steps)

30

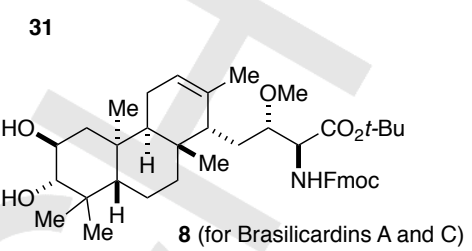

b<smiles>CCCC1(C)C(C)CC=C(C)[C@H]1C[C@H](O)[C@H](O[N+](=O)[O-])C(=O)OC(C)(C)C</smiles>

32

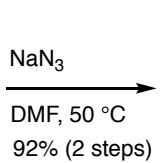<smiles>CCCC1(CC)C(C)CC=C([N+](=O)[O-])C1CC(O)C(N)C(=O)OC(C)(C)C</smiles>

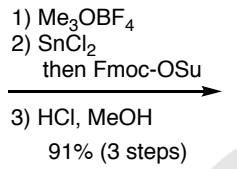

1) $\mathrm{Me}_{3} \mathrm{OBF}_{4}$
$\underset{\text { 3hen } \mathrm{Fmoc}-\mathrm{OSu}}{\mathrm{HCl}, \mathrm{MeOH}}$
$\underset{91 \% \text { (3 steps) }}{\longrightarrow}$

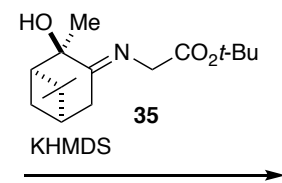

THF, $-65^{\circ} \mathrm{C}$

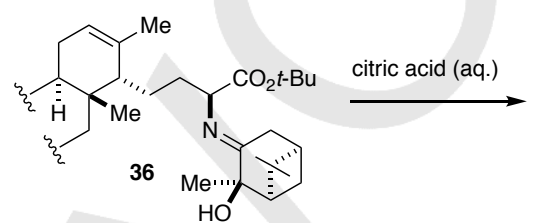

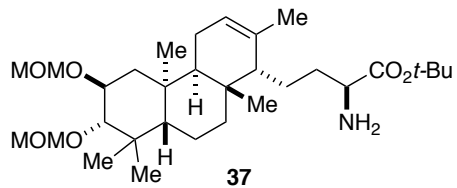

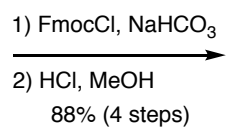

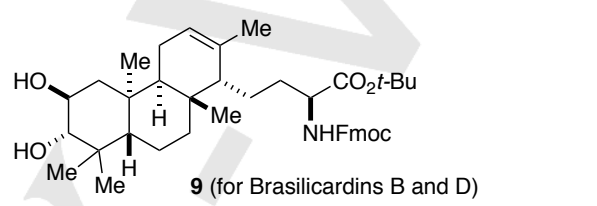

Scheme 4. Stereoselective synthesis of the protected brasilicardin aglycons 8 and $9 . \quad p-N s=4-$ itrobenzenesulfonyl, Su $=$ succinimidyl, Fmoc $=9$ fluorenylmethyloxycarbonyl, KHMDS = potassium bis(trimethylsilyl)amide.

glycosylation product and degradation of $\mathbf{8}$ were observed. To our delight, after extensive screening of glycosyl donors and their activation conditions, the metallocene-based activator $\mathrm{Cp}_{2} \mathrm{HfCl}_{2} / \mathrm{AgOTf}{ }^{27]}$ allowed the regio- and stereoselective coupling of peracetyl glycosyl fluoride $\mathbf{3 8}$ (2 equiv) with $\mathbf{8}$ to give the desired $\alpha$-glycoside 39 (with $43 \%$ of 8 recovered). Because the prolonged reaction time caused an undesired reaction at the C3 alcohol, this coupling was carefully stopped before full consumption of $\mathbf{8}$, with recovery of unreacted $\mathbf{8}$. Finally, removal of the tert-butyl ester using TFA followed by global deprotection of the acetyl and Fmoc groups furnished brasilicardin A (1) $(43 \%$ yield, three steps). Confirming the identity of our synthesized sample was challenging because the ${ }^{1} \mathrm{H}-\mathrm{NMR}$ spectrum of the compound proved highly dependent on the concentration and $\mathrm{pH}$. However, its identification was achieved using ${ }^{1} \mathrm{H}$-NMR spectrum of a mixture of synthetic and natural brasilicardin $A$ in a 1:1 ratio after both materials were purified by reverse phase semipreparative HPLC (Capcell Pak C18 SG120). Further, the fact that the ${ }^{13} \mathrm{C}-\mathrm{NMR}, \mathrm{IR}$, and HRMS spectra and optical rotation results for the synthesized product matched those of the natural sample supported successful synthesis of our desired product, as did the narrow range obtained for the melting temperature of the mixture. Brasilicardin B (2) was also synthesized from 9 using the same conditions as those used for 1 .

Achievement of total synthesis of $\mathbf{1}$ and $\mathbf{2}$ prompted us to pursue the total synthesis of brasilicardins $C(3)$ and $D(4)$ (Scheme 5b). Unlike the glycosylation with disaccharide $\mathbf{3 8}$, glycosylation of the glycosyl fluoride donor derived from rhamnose with diols $\mathbf{8}$ or $\mathbf{9}$ did not show any regioselectivity at the C2 alcohol. Attempted use of other donors also failed.
Consequently, the $\mathrm{C} 3$ alcohol was temporary protected as an easily removable methoxyacetate ${ }^{[28]}$ and the requisite monoalcohols $\mathbf{4 1}$ and $\mathbf{4 2}$ were synthesized from $\mathbf{8}$ and $\mathbf{9}$, respectively, through a three-step conversion. Toward the synthesis of total synthesis of brasilicardin $\mathrm{C}$, we found that gold-catalyzed coupling $^{[29]}$ of glycosyl o-cyclopropylethynylbenzoate $\mathbf{4 3}$ with $\mathbf{4 1}$ proved to be the most effective method for stereoselective installation of a rhamnose, providing the glycosylation product $4 \mathbf{4}$. This compound was successfully converted to brasilicardin C (3) via TFA-mediated removal of tert-butyl ester and the subsequent global deprotection of the remaining acetyl, methoxyacetyl, and Fmoc groups with aqueous lithium hydroxide solution. In addition, brasilicardin $D$ (4) was obtained from $\mathbf{4 2}$ via a similar sequence as that described for 3 . Thus, we accomplished the first total synthesis of brasilicardin B (2) and brasilicardin D (4) in addition to the total synthesis of brasilicardin A (1) and brasilicardin C (3).

In conclusion, we have developed a stereoselective synthetic route to synthesize brasilicardins with potent immunosuppressive activity, utilizing which we accomplished the asymmetric total synthesis of brasilicardin A (39 linear steps, $6.8 \%$ yield), brasilicardin B (37 linear steps, $6.5 \%$ yield), brasilicardin C (42 linear steps, $12 \%$ yield), and brasilicardin D (40 linear steps, $14 \%$ yield) from readily available commercial materials. The synthesis features (1) the development of a novel intramolecular nitrile Michael addition, i.e., stereoselective intramolecular conjugate addition of an $\alpha$-cyano carbanion to an $\alpha, \beta$-unsaturated Weinreb amide, which allows simultaneous ring formation and construction of contiguous quaternary-tertiary asymmetric stereocenters, (2) a Michael addition-based strategy for the stereoselective formation of the highly strained 
a

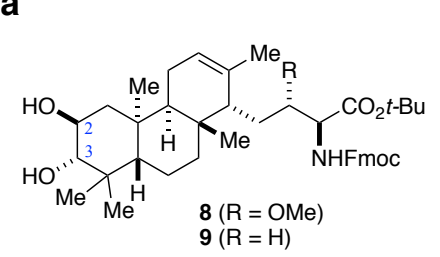

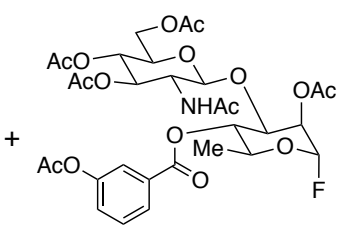

38 ( $\alpha$-anomer exclusively)

$$
\text { 1) TFA }
$$

2) ethylenediamine, EtOH

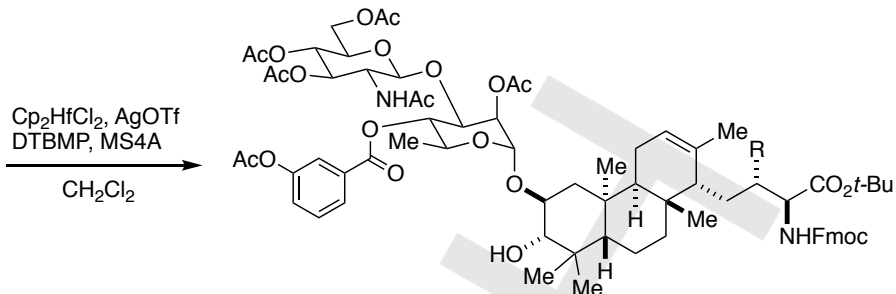

$39(\mathrm{R}=\mathrm{OMe})+$ recovery of $8(43 \%)$ $40(\mathrm{R}=\mathrm{H})+$ recovery of $9(61 \%)$

b
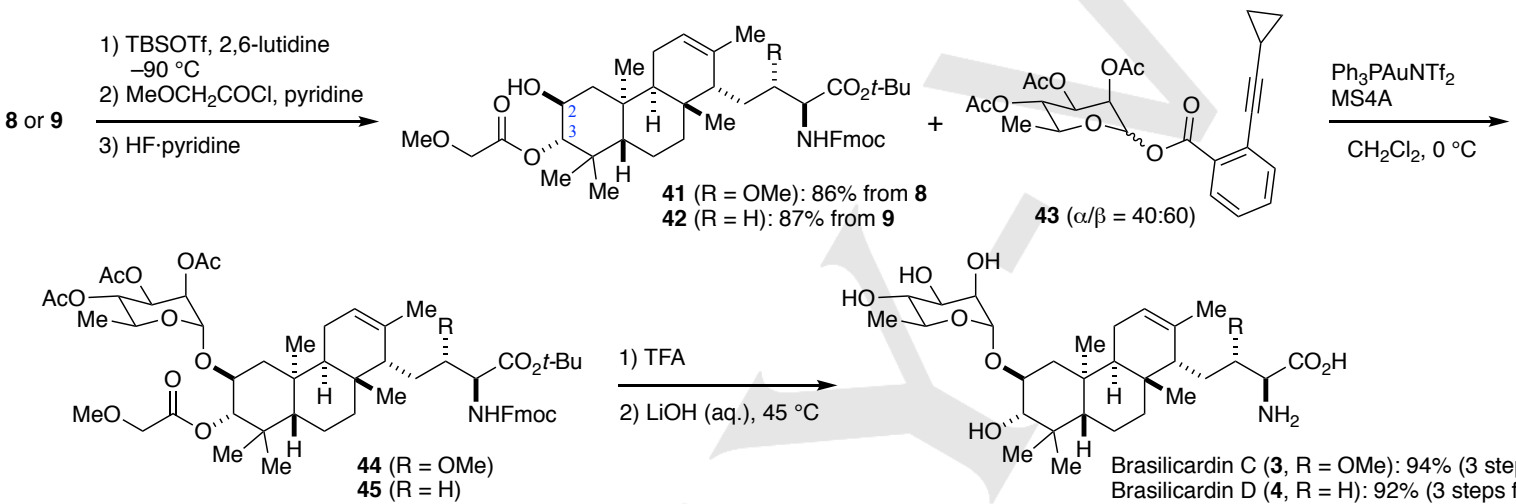

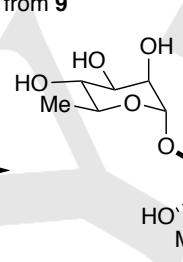

$43(\alpha / \beta=40: 60)$

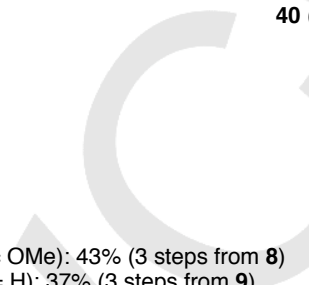

Brasilicardin $A(1, R=O M e): 43 \%$ (3 steps from 8 )

$45(\mathrm{R}=\mathrm{H})$

Scheme 5. Total synthesis of brasilicardins A-D. DTBMP $=2,6$-di-tert-butyl-4-methylpyridine, MS4A $=$ molecular sieves $4 \mathrm{~A}$, TFA $=$ trifluoroacetic acid. Tf $=$ trifluoromethanesulfonyl.

anti-syn-anti-fused perhydrophenanthrene skeleton (the ABCring), (3) stereoselective installation of the amino acid component to the terpenoid core, and (4) regio- and stereoselective glycosylation using glycosyl fluoride or 0 alkynylbenzoate as the glycosyl donor. Our strategy allowed the unified synthesis of all brasilicardins from the common late-stage intermediate utilizing the appropriate installation methods for an amino acid and glycosylation. The novel synthetic route developed here would accelerate the synthesis and biological studies of brasilicardins and a wide variety of their analogues that were previously inaccessible by syntheses or from natural products as well as aid in obtaining in-depth SAR toward the development of new immunosuppressive drugs.

\section{Acknowledgements}

We acknowledge gratitude toward Professors Jun'ichi Kobayashi (Hokkaido University) and Takaaki Kubota (Showa Pharmaceutical University) for providing an authentic sample of brasilicardin A. The authors thank Professor Hideyuki Shigemori (University of Tsukuba) and Dr. Hisayuki Komaki (NBRC) for helpful discussions regarding the purification protocols of brasilicardins. Additionally, we acknowledge help from Dr. Eri
Fukushi and Mr. Yusuke Takata (GC-MS \& NMR Laboratory, Faculty of Agriculture, Hokkaido University) in performing mass spectral measurements, Dr. Yasuhiro Kumaki (High-Resolution NMR Laboratory, Graduate School of Science, Hokkaido University) for the NMR experiments, and Professors Tamotsu Inabe and Takahiro Suzuki (Hokkaido University) for the X-ray structural analysis. This work was supported by JSPS KAKENHI Grant Numbers JP22710206, JP24510290, JP15K01795, JP18K05339, JP18H01970, and JP15H05842 in Middle Molecular Strategy.

Keywords: Total synthesis $•$ Brasilicardins $•$ Natural products $•$ Michael addition $\bullet$ Quaternary stereocenters

[1] H. Shigemori, H. Komaki, K. Yazawa, Y. Mikami, A. Nemoto, Y. Tanaka, T. Sasaki, Y. In, T. Ishida, J. Kobayashi, J. Org. Chem. 1998, 63, 69006904.

[2] K. Komatsu, M. Tsuda, M. Shiro, Y. Tanaka, Y. Mikami, J. Kobayashi, Bioorg. Med. Chem. 2004, 12, 5545-5551.

[3] H. Komaki, A. Nemoto, Y. Tanaka, H. Takagi, K. Yazawa, Y. Mikami, H. Shigemori, J. Kobayashi, A. Ando, Y. Nagata, J. Antibiot. 1999, 52, 1319.

[4] T. Usui, Y. Nagumo, A. Watanabe, T. Kubota, K. Komatsu, J. Kobayashi, H. Osada, Chem. Biol. 2006, 13, 1153-1160. 
[5] K. Komatsu, M. Tsuda, Y. Tanaka, Y. Mikami, J. Kobayashi, Bioorg. Med. Chem. 2005, 13, 1507-1513.

[6] M. E. Jung, B. T. Chamberlain, P. Koch, K. R. Niazi, Org. Lett. 2015, 17, 3608-3611.

[7] D. M. Coltart, S. J. Danishefsky, Org. Lett. 2003, 5, 1289-1292.

[8] M. E. Jung, P. Koch, Org. Lett. 2011, 13, 3710-3713.

[9] M. E. Jung, F. Perez, C. F. Regan, S. W. Yi, Q. Perron, Angew. Chem. Int. Ed. 2013, 52, 2060-2062; Angew. Chem. 2013, 125, 2114-2116.

[10] M. Anada, T. Hanari, K. Kakita, Y. Kurosaki, K. Katsuse, Y. Sunadoi, Y. Jinushi, K. Takeda, S. Matsunaga, S. Hashimoto, Org. Lett. 2017, 19, 5581-5584.

[11] M. Nishizawa, H. Takenaka, K. Hirotsu, T. Higuchi, Y. Hayashi, J. Am. Chem. Soc. 1984, 106, 4290-4291.

[12] S. Balasubramaniam, I. S. Aidhen, Synthesis 2008, 3707-3738.

[13] K. M. Byrd, Beilstein J. Org. Chem. 2015, 11, 530-562.

[14] S. Arseniyadis, K. S. Kyler, D. S. Watt, Org. React. 1984, 31, 1-364.

[15] F. Yoshimura, M. Torizuka, G. Mori, K. Tanino, Synlett 2012, 23, 251254.

[16] K. Tanino, K. Arakawa, M. Satoh, Y. Iwata, M. Miyashita, Tetrahedron Lett. 2006, 47, 861-864.

[17] M. A. Blanchette, W. Choy, J. T. Davis, A. P. Essenfeld, S. Masamune, W. R. Roush, T. Sakai, Tetrahedron Lett. 1984, 25, 2183-2186.

[18] K. B. Sharpless, W. Amberg, M. Beller, H. Chen, J. Hartung, Y. Kawanami, D. Lübben, E. Manoury, Y. Ogino, T. Shibata, T. Ukita, J. Org. Chem. 1991, 56, 4585-4588.

[19] K. Ohmori, S. Nishiyama, S. Yamamura, Tetrahedron Lett. 1995, 36, 6519-6522.

[20] B. Gasper, E. M. Carreira, Angew. Chem. Int. Ed. 2007, 46, 4519-4522; Angew. Chem. 2007, 119, 4603-4606.

[21] K. Ando, J. Org. Chem. 1997, 62, 1934-1939.

[22] F. P. Touchard, N. Capelle, M. Mercier, Adv. Synth. Catal. 2005, 347, 707-711.

[23] O. Labeeuw, P. Phansavath, J.-P. Genêt, Tetrahedron Lett. 2004, 45, 7107-7110.

[24] A. V. Rama Rao, T. K. Chakraborty, K. Laxma Reddy, A. Srinivasa Rao, Tetrahedron Lett. 1994, 35, 5043-5046.

[25] S. Yamada, T. Oguri, T. Shioiri, J. Chem. Soc., Chem. Commun. 1976 , 136-137.

[26] I. Ohtani, T. Kusumi, Y. Kashman, H. Kakisawa, J. Am. Chem. Soc. 1991, 113, 4092-4096.

[27] K. Suzuki, H. Maeta, T. Matsumoto, Tetrahedron Lett. 1989, 30, 48534856.

[28] C. B. Reese, J. C. M. Stewart, J. H. van Boom, H. P. M. de Leeuw, J. Nagel, J. F. M. de Rooy, J. Chem. Soc., Perkin Trans. 11975, 934-942.

[29] B. Yu, Acc. Chem. Res. 2018, 51, 507-516.

[30] CCDC 1550053, 1551801, 1566258 (for compounds 10, 28, and $p$ bromobenzamide derivative of $\mathbf{8}$ (i.e., compound $\mathbf{S} 2$ in the Supporting Information)) contain the supplementary crystallographic data for this paper. These can be obtained free of charge from The Cambridge Crystallographic Data Centre. 
Entry for the Table of Contents (Please choose one layout)

\section{COMMUNICATION}

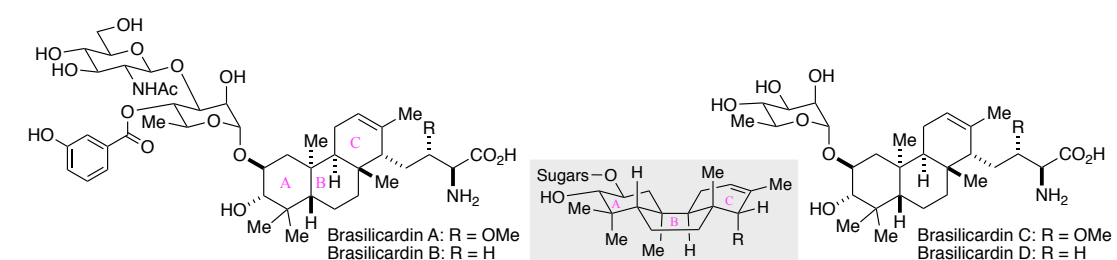

The asymmetric total synthesis of brasilicardin $\mathrm{A}$, a hybrid natural product with highly potent immunosuppressive activity, has been developed. The synthesis features a Michael addition-based strategy for the stereoselective formation of the highly strained anti-syn-anti-fused perhydrophenanthrene skeleton, and brasilicardins B-D are also synthesized based on the unified approach.
Fumihiko Yoshimura, ${ }^{*}$ Ryusei Itoh, Makoto Torizuka, Genki Mori, Keiji Tanino*

\section{Page No. - Page No.}

Asymmetric Total Synthesis of Brasilicardins 